\title{
Phase I and II Vaccine Trials
}

National Cancer Institute

\section{Source}

National Cancer Institute. Phase I and II Vaccine Trials. NCI Thesaurus. Code C15835.

Select candidate vaccines or concepts suitable for Phase I and Phase II trials and conduct these trials. 